J. DIFFERENTIAL GEOMETRY

62 (2002) 49-78

\title{
ON THE ASYMPTOTIC ISOPERIMETRIC CONSTANTS FOR RIEMANN SURFACES AND GRAPHS
}

\author{
ROBERT BROOKS \& ANDRZEJ ZUK
}

\begin{abstract}
We study the behavior of the Cheeger isoperimetric constant on infinite families of graphs and Riemann surfaces, and its relationship to the first eigenvalue $\lambda_{1}$ of the Laplacian. We adapt probabilistic arguments of Bollobás to the setting of Riemann surfaces, and then show that Cheeger constants of the modular surfaces are uniformly bounded from above away from the maximum value. We extend this result to the class of Ramanujan surfaces, defined below.
\end{abstract}

\section{Introduction}

To a manifold or a graph one can associate two numbers which carry interesting geometric and analytic information, namely the Cheeger isoperimetric constant and the bottom of the $L^{2}$ spectrum of the Laplace operator. Their study has a long history, and many results concerning relations between these two quantities have been obtained.

For a connected regular graph of degree $k$, let $\Delta$ be the discrete Laplace operator, acting on $L^{2}(X)$ by the formula

$$
\Delta f(x)=f(x)-\frac{1}{k} \sum_{y \sim x} f(y),
$$

where $f \in L^{2}(X), x$ is a vertex of $X$ and " $y \sim x$ " means that $y$ and $x$ are connected by an edge. The operator $\Delta$ is self-adjoint and nonnegative.

The first author was partially supported by the Israel Science Foundation and the Fund for the Promotion of Research at the Technion.

Received 05/23/2002. 
For an infinite graph $X$, let $\lambda_{0}(X)$ denote the bottom of the $L^{2}$ spectrum of $\Delta$. For a finite graph $X$, let $\lambda_{1}(X)$ denote the first nonzero eigenvalue of $\Delta$.

The asymptotic behavior of the first nonzero eigenvalue $\lambda_{1}$ of the Laplace operator on finite graphs has been studied extensively. In particular, the theorem of Alon-Boppana states:

Proposition 1.1 ([1], [15]). Let $X$ be a finite $k$-regular graph, where $k$ is fixed. Then

$$
\lambda_{1}(X) \leq 1-\frac{2 \sqrt{k-1}}{k}+\varepsilon(|X|)
$$

where $\varepsilon(|X|) \rightarrow 0$ as $|X| \rightarrow \infty$.

We introduce here a convention which will be in force throughout this paper: the function $\varepsilon\left(x_{1}, \ldots, x_{k}\right)$ will denote a function of the variables $x_{1}, \ldots, x_{k}$ which tends to 0 as the variables tend to infinity.

The number $1-\frac{2 \sqrt{k-1}}{k}$ is the bottom $\lambda_{0}\left(T_{k}\right)$ of the $L^{2}$ spectrum of the Laplace operator on the regular tree $T_{k}$ of degree $k$, which is the universal cover of the graph $X$.

Results of Lubotzky, Phillips, and Sarnak [15] and Margulis [17] show that for infinitely many values of $k$, the bound $1-\frac{2 \sqrt{k-1}}{k}+\varepsilon(|X|)$ in Proposition 1.1 is optimal.

We begin this paper by studying the asymptotic behavior of the Cheeger isoperimetric constant of a $k$-regular graph.

Let $X$ be a finite graph of degree $k$. For a finite subset of vertices $U \subset X$ we define its boundary $\partial U$ as the set of edges with one extremity in $U$ and the other in $X \backslash U$. We define the Cheeger isoperimetric constant $h(X)$ as

$$
h(X)=\min \left\{\frac{|\partial U|}{|U|} ; U \subset X \text { and } 1 \leq|U| \leq \frac{1}{2}|X|\right\} .
$$

There are a number of relations between the Cheeger constant $h(X)$ and the eigenvalues $\lambda_{0}(X)$ and $\lambda_{1}(X)$ of $\Delta$ acting on $L^{2}(X)$, see Propositions 4.1 and 4.2 below.

Extending a result of Bollobás [3], we show that the asymptotic behavior of the isoperimetric constant of a finite $k$-regular graph behaves differently than the first eigenvalue of the Laplacian with respect to universal covering (compare [2]). 
Let $\operatorname{inj}(X)$ denote the injectivity radius of the graph $X$, i.e., the maximal number $r$ such that in every ball of radius $r$ in $X$ there is no cycle. The girth is related to the injectivity radius by the formula $\operatorname{inj}(X)=\left\lceil\frac{\operatorname{girth}(X)}{2}\right\rceil$.

Theorem 3.1. Let $X$ be a finite $k$-regular graph. Then

$$
h(X) \leq \frac{k-2}{2}+\varepsilon(\operatorname{inj}(X))=\frac{h\left(T_{k}\right)}{2}+\varepsilon(\operatorname{inj}(X)) .
$$

A result stronger than Theorem 3.1 is contained in [2]. Indeed, in [2] the assumption on injectivity radius is not needed, and one obtains a constant of the form $\frac{k}{2}-c \sqrt{k}$, where $c$ does not depend on $k$. We remark that

$$
\frac{(k / 2)-c \sqrt{k}}{(1 / 2)\left(h\left(T_{k}\right)\right)} \rightarrow 1 \text { as } k \rightarrow \infty .
$$

We present Theorem 3.1 with the weaker assumption and weaker conclusion, as the dependence on injectivity radius and universal covering will reappear in the geometric setting, Theorem 5.1 below.

We now turn to the problem of generalizing Theorem 3.1 to the setting of manifolds, and in particular to the case of Riemann surfaces.

For a Riemannian manifold $M^{n}$ of dimension $n$ we define its Cheeger isoperimetric constant $h\left(M^{n}\right)$ as follows

$$
h\left(M^{n}\right)=\inf _{A}\left\{\frac{\operatorname{vol}_{n-1}(\partial A)}{\operatorname{vol}_{n}(A)}\right\}
$$

where $A$ runs over subdomains of $M^{n}$ of volume less than $(1 / 2) \operatorname{vol}\left(M^{n}\right)$, and where $\operatorname{vol}_{n-1}(\partial A)$ and $\operatorname{vol}_{n}(A)$ are the measures with respect to the Riemannian metric.

It is well-known that for the hyperbolic plane $\mathbb{H}^{2}$ one has

$$
h\left(\mathbb{H}^{2}\right)=1 .
$$

Let $\operatorname{inj}(M)$ denote the injectivity radius of $M$. When $M$ has negative curvature, then $\operatorname{inj}(M)$ is the largest number $r$ such that every ball of radius $r$ in $M$ is isometric to its lift in the universal cover $\widetilde{M}$ of $M$. Thus, $\operatorname{inj}(M)$ is in this case equal to half the length of the shortest closed geodesic on $M$. 
We show in Theorem 5.1 below that if $S$ is a Riemann surface of genus $>1$, endowed with its hyperbolic metric, then, modulo some technical assumptions that will be explained there, we have

$$
h(S) \leq(1 / 2)+\varepsilon(\operatorname{inj}(S))=(1 / 2)\left[h\left(\mathbb{H}^{2}\right)+\varepsilon(\operatorname{inj}(S))\right] .
$$

We then show the following theorem in Section 6 :

Theorem 6.2. There is a constant $C<1 / 2$ with the following property:

Let $S_{k}$ be the modular surface

$$
S_{k}=\mathbb{H}^{2} / \Gamma_{k},
$$

where $\Gamma_{k}$ is the subgroup of $\operatorname{PSL}(2, \mathbb{Z})$ given by

$$
\Gamma_{k}=\left\{\left(\begin{array}{ll}
a & b \\
c & d
\end{array}\right):\left(\begin{array}{ll}
a & b \\
c & d
\end{array}\right) \equiv\left(\begin{array}{ll}
1 & 0 \\
0 & 1
\end{array}\right)(\bmod k)\right\} .
$$

Then

$$
h\left(S_{k}\right) \leq C
$$

for $k$ sufficiently large.

Our argument gives the explicit bound

$$
C \leq .4402 \ldots
$$

The modular surfaces $S_{k}$ were studied by Selberg [20], who proved that the first eigenvalue $\lambda_{1}\left(S_{k}\right)$ satisfies

$$
\lambda_{1}\left(S_{k}\right) \geq 3 / 16 \text {. }
$$

He also conjectured that one had the stronger bound

$$
\lambda_{1}\left(S_{k}\right) \geq 1 / 4
$$

where $1 / 4$ is the bottom of the spectrum of $\mathbb{H}^{2}$. In light of Theorem 5.1, one can think of Theorem 6.2 as saying that the analogue of the conjecture of Selberg in the context of the Cheeger constant is false.

A common theme in the proofs of Theorems 3.1 and 5.1 is the use of probabilistic arguments originally introduced by Bollobás in [3]. Basic facts from probability theory which we use are recalled in Section 2. 
In Section 7, we extend Theorem 6.2 to surfaces built out of the Ramanujan graphs of [15]. The construction describing how these surfaces are built from $k$-regular graphs is sketched in Section 7 . We then show:

Theorem 7.1. There exists a constant $C<1 / 2$ such that, for all $p$ and for all $q$ sufficiently large, depending on $p$, we have that

$$
h\left(S_{\mathcal{O}}^{p, q}\right) \leq C,
$$

where $S_{\mathcal{O}}^{p, q}$ is a surface constructed from the Ramanujan graph $X^{p, q}$.

Our argument produces a bound for $C$ of

$$
C \leq .467177 \ldots
$$

We remark that in contrast to the graph-theoretic results of [2], we have that this constant stays away from $1 / 2$ independent of the regularity $k$ of the associated graph.

Acknowledgements. The first author would like to thank the École Normale Superieure of Lyon and the University of Chicago for their hospitality while much of this research was carried out.

The second author would like to thank the Technion for its hospitality during a visit where this research was begun.

After a preliminary version of this paper was completed, Noga Alon informed us of his work [2]. In addition to proving results stronger than the graph theoretic results contained in this paper, he also made use of very similar techniques. We would like to thank him for bringing our attention to [2].

\section{Probability}

In our considerations below, we make use of some basic, well-known facts from probability theory. Because they are usually not used in the geometric context, we will recall them.

Let $C_{n}^{\frac{n}{2}}$ denote the set of all subsets of $\{1, \ldots, n\}$ of size $\frac{n}{2}$.

Lemma 2.1. Let $r<R$ be fixed positive numbers. Let $r_{1}, \ldots, r_{n}$ be such that $r \leq r_{i} \leq R$. Then for any $\varepsilon>0$

$$
\begin{aligned}
& \frac{\#\left\{I \in C_{n}^{\frac{n}{2}} ;\left(\frac{1}{2}-\varepsilon\right) \sum_{i=1}^{n} r_{i} \leq \sum_{i \in I} r_{i} \leq\left(\frac{1}{2}+\varepsilon\right) \sum_{i=1}^{n} r_{i}\right\}}{\# C_{n}^{\frac{n}{2}}} \\
& \geq 1-F(r, R, \varepsilon, n),
\end{aligned}
$$


where $F(r, R, \varepsilon, n)$ tends to 0 when $n$ tends to infinity.

Proof. Let $G_{n}^{\frac{n}{2}}$ denote these subsets $I$ of $C_{n}^{\frac{n}{2}}$ for which we have

$$
\left(\frac{1}{2}-\varepsilon\right) \sum_{i=1}^{n} r_{n} \leq \sum_{i \in I} r_{i} \leq\left(\frac{1}{2}+\varepsilon\right) \sum_{i=1}^{n} r_{i}
$$

We want to prove that

$$
\frac{\# G_{n}^{\frac{n}{2}}}{\# C_{n}^{\frac{n}{2}}} \geq 1-F(r, R, \varepsilon, n)
$$

where $F(r, R, \varepsilon, n)$ tends to 0 when $n$ tends to infinity.

Let us consider two numbers $r_{i}$ and $r_{j}$ from the sequence $r_{1}, \ldots, r_{n}$ such that $r_{i} \leq r_{j}$. For any $\delta>0$ let us consider instead of $r_{i}, r_{j}$ the numbers $r_{i}^{\prime}=r_{i}-\delta$ and $r_{j}^{\prime}=r_{j}+\delta$. The sum of the numbers did not change. Let $G_{n}^{\prime \frac{n}{2}}$ denote these subsets $I$ of $C_{n}^{\frac{n}{2}}$ which satisfy (1) for a new sequence of $r$ 's where $r_{i}, r_{j}$ are replaced by $r_{i}^{\prime}, r_{j}^{\prime}$ respectively. The cardinality of $G^{\prime \frac{n}{2}}$ can only decrease with respect to the cardinality of $G_{n}^{\frac{n}{2}}$. Indeed, whenever we have $I \subset G_{n}^{\frac{n}{2}}$ also its complement $I^{\prime}$ in $\{1, \ldots, n\}$ belongs to $G_{n}^{\frac{n}{2}}$. As $r_{i} \leq r_{j}$ we will have more $I^{\prime} \subset G_{n}^{\prime \frac{n}{2}}$ for which

$$
\sum_{i \in I^{\prime}} r_{i}<\left(\frac{1}{2}-\varepsilon\right) \sum_{i=1}^{n} r_{i}
$$

or

$$
\sum_{i \in I^{\prime}} r_{i}>\left(\frac{1}{2}+\varepsilon\right) \sum_{i=1}^{n} r_{i}
$$

then $I \subset G_{n}^{\frac{n}{2}}$ with the same property.

After applying this procedure several times we obtain the following situation: $r_{1}, \ldots, r_{k}=r$ and $r_{k+1}, \ldots, r_{n}=R$ or $r_{1}, \ldots, r_{k}=r$ and $r_{k+1}, \ldots, r_{n-1}=R$ and $r_{1}, \ldots, r_{k}=r$ and $r_{k+1}, \ldots, r_{n-1}=R$ and $r_{n}=c$, where $r<c<R$ and we need to prove Lemma 2.1 for such a situation. But in this case the statement of Lemma 2.1 is a consequence of the Law of Large Numbers. q.e.d. 


\section{Asymptotic isoperimetric constants for graphs}

A graph $X$ is said to be $k$-regular if every vertex meets exactly $k$ edges.

Let $X$ be a finite $k$-regular graph. For a finite subset of vertices $U \subset X$ we define its boundary $\partial U$ as the set of edges with one extremity in $U$ and another one in $X \backslash U$. We define the Cheeger isoperimetric constant $h(X)$ as

$$
h(X)=\min \left\{\frac{|\partial U|}{|U|} ; U \subset X \text { and } 1 \leq|U| \leq \frac{1}{2}|X|\right\} .
$$

Proposition 3.1 (Bollobás [3]). Let X be a finite $k$-regular graph. Then

$$
h(X) \leq \frac{k}{2}+\varepsilon(|X|) .
$$

One can also define the isoperimetric constant in the same way as before for graphs which are not of constant degree. Then one can prove

Proposition 3.2. Let $X$ be a connected graph with $E$ edges and $n$ vertices. Then there exists a subset $A_{0}$ such that $\left|A_{0}\right|=[n / 2]$ and

$$
\frac{\left|\partial A_{0}\right|}{\left|A_{0}\right|} \leq E \frac{n+1}{(n-1) n} .
$$

Proof. Let $A$ be a random subset of $X$, such that $|A|=\left[\frac{n}{2}\right]$. Let us compute the expected number of edges of $X$ with one end in $A$ and another one in $A^{c}$. If $X$ had just one edge then the expected number would be equal to

$$
\frac{|A|(n-|A|)}{\left(\begin{array}{c}
n \\
2
\end{array}\right)} .
$$

As $X$ has $E$ edges, the expected number is equal

$$
E \frac{|A|(n-|A|)}{\left(\begin{array}{c}
n \\
2
\end{array}\right)} .
$$

Thus there exists $A_{0} \subset X$ of cardinality $\left[\frac{n}{2}\right]$ such that

$$
\left|\partial A_{0}\right| \leq E \frac{\left|A_{0}\right|\left(n-\left|A_{0}\right|\right)}{\left(\begin{array}{c}
n \\
2
\end{array}\right)} .
$$


This gives:

$$
\frac{\left|\partial A_{0}\right|}{\left|A_{0}\right|} \leq E \frac{\left(n-\left|A_{0}\right|\right)}{\left(\begin{array}{c}
n \\
2
\end{array}\right)}=E \frac{\left(n-\left[\frac{n}{2}\right]\right)}{\left(\begin{array}{c}
n \\
2
\end{array}\right)} \leq E \frac{(n+1)}{(n-1) n} .
$$

This ends the proof of Proposition 3.2.

q.e.d.

Corollary 3.1. Let $k$ be fixed. Let $X$ be a connected graph with $\frac{k|X|}{2}$ edges. Then

$$
h(X) \leq \frac{k}{2}+\varepsilon(|X|) .
$$

If the graph $X$ is infinite, we define the Cheeger isoperimetric constant $h(X)$ as

$$
h(X)=\min _{U}\left\{\frac{|\partial U|}{|U|}\right\},
$$

where $U$ ranges over finite subsets of $X$.

We will say that a sequence of graphs $X_{i}$ converges to a connected graph $X$, i.e., $X_{i} \rightarrow_{i \rightarrow \infty} X$, if there exists a vertex $x \in X$ and a sequence of natural numbers $r_{i} \in \mathbb{N}$ such that $r_{i} \rightarrow_{i \rightarrow \infty} \infty$ and the balls $B\left(x_{i}, r_{i}\right)$ and $B\left(x, r_{i}\right)$ are isometric. The limit graph is not unique and may depend on the choice of the sequence $x_{i}$.

Proposition 3.3. Let $X_{i}$ be a sequence of finite or infinite, connected graphs, such that $X_{i} \rightarrow_{i \rightarrow+\infty} X$ where the graph $X$ is infinite and connected. Then

$$
\limsup _{i \rightarrow+\infty} h\left(X_{i}\right) \leq h(X)
$$

Proof. This is clear from the definition of convergence.

q.e.d.

Let $T_{k}$ be the regular tree of degree $k$. It is well-known that

$$
h\left(T_{k}\right)=k-2 .
$$

For any regular infinite graph $X$ of degree $k$ we then have that

$$
h(X) \leq h\left(T_{k}\right)=k-2 .
$$

Let $\operatorname{inj}(X)$ denote the injectivity radius of the graph $X$. 
Theorem 3.1. Let $X$ be a finite $k$-regular graph.

Then

$$
h(X) \leq \frac{k-2}{2}+\varepsilon(\operatorname{inj}(X))=\frac{h\left(T_{k}\right)}{2}+\varepsilon(\operatorname{inj}(X)) .
$$

Proof. First of all, we need the following

Lemma 3.1. Let $A$ be a finite connected subset of a regular tree $T_{k}$ of degree $k$. Then

$$
k-2 \leq \frac{|\partial A|}{|A|} \leq \varepsilon(\operatorname{diam}(A)) .
$$

Proof. Because the isoperimetric constant of a regular tree of degree $k$ is $k-2$, we have $\frac{|\partial A|}{|A|} \geq k-2$.

If $A$ is an interval of $\operatorname{diam}(A)$, we have that

$$
|A|=\operatorname{diam}(A)+1
$$

while

$$
|\partial A|=2(k-1)+(\operatorname{diam}(A))(k-2),
$$

so that

$$
\frac{|\partial A|}{|A|}=(k-2)+\frac{2}{\operatorname{diam}(|A|)+1}=k-2+\varepsilon(\operatorname{diam}(A)) .
$$

If $A$ is not an interval, then there exists some vertex $v$ whose boundary contains $k-1$ edges. Removing this vertex and the edge joining it to the rest of $A$ decreases the edge set by $k-2$ and decreases the vertex set by 1 , and doesn't change the diameter. Since $\frac{|\partial A|}{|A|} \geq k-2$, this process increases the isoperimetric ratio. Continuing this process until we get an interval establishes the lemma.

q.e.d.

We remark that the lower bound of Lemma 3.1 is not sharp. Indeed, the argument shows that the infimum is attained by the "ball of diameter $\operatorname{diam}(A)$, which for $\operatorname{diam}(A)$ even is the ball of radius $\frac{\operatorname{diam}(A)}{2}$, and for $\operatorname{diam}(A)$ odd is the union of the two balls of radius $\frac{\operatorname{diam}(A)-1}{2}$ about the two endpoints of an edge.

We now return to the proof of Theorem 3.1.

Let $R$ be the injectivity radius of the graph $X$. In the graph $X$, let us consider a maximal set of vertices $v_{1}, \ldots, v_{n}$ such that any two vertices are at distance at least $R / 10$. 
Let us consider disjoint subsets $C_{1}, \ldots, C_{n}$ of $X$ with the property that if $v \in C_{i}$, then

$$
\operatorname{dist}\left(v, v_{j}\right) \geq \operatorname{dist}\left(v, v_{i}\right) \quad \text { for all } j \text {. }
$$

We will need some simple properties of the sets $C_{i}$ :

Lemma 3.2. The sets $C_{i}$ have the following properties:

(i) $\frac{R}{10} \leq \operatorname{diam}\left(C_{i}\right) \leq \frac{R}{5}$.

(ii) Each $C_{i}$ is a connected subgraph of $T_{k}$.

(iii) For $i \neq j$, there exists at most one edge conecting a vertex of $C_{i}$ with a vertex of $C_{j}$.

(iv) The number $n$ of the $C_{i}$ 's is bounded below by a function of $R$ which goes to infinity as $R \rightarrow \infty$.

We now consider the graph $V$ which has one vertex $v_{i}$ for each $C_{i}$, and where $v_{i}$ is joined by an edge to $v_{j}$ if there is an edge joining a vertex of $C_{i}$ to a vertex of $C_{j}$.

Because the subset $A$ was chosen at random, by Lemma 2.1 we can assume, by taking if necessary instead of $A$ its complement, that

$$
\frac{1}{2}-\varepsilon(n) \leq \frac{\left|C_{i_{1}}\right|+\cdots+\left|C_{i_{\frac{n}{2}}}\right|}{\left|C_{1}\right|+\cdots+\left|C_{n}\right|} \leq \frac{1}{2},
$$

where $\varepsilon(n) \rightarrow_{n \rightarrow \infty} 0$. For a subset $C \subset X_{i}$ such that

$$
C=C_{i_{1}} \cup \cdots \cup C_{i_{\frac{n}{2}}}
$$

we have $|C| \leq\left|X_{i}\right| / 2$ and

$$
|\partial C|=|\partial A| .
$$

Because $C_{1}, \ldots, C_{n}$ are subsets of $T_{k}$ and we have by Lemma 3.1 that

$$
\left|\partial C_{i}\right| \leq(k-2+\varepsilon(R))\left|C_{i}\right|
$$


where $\varepsilon(R)$ tends to 0 when $R$ tends to infinity. Thus

$$
\begin{aligned}
\frac{|\partial C|}{|C|} & =\frac{|\partial A|}{\left|C_{i_{1}}\right|+\cdots+\left|C_{i_{\frac{n}{2}}}\right|} \\
& \leq \frac{E|A|(n+2)}{(n-1) n\left(\left|C_{i_{1}}\right|+\cdots+\left|C_{i_{\frac{n}{2}}}\right|\right)} \\
& =\frac{1}{4}\left(\frac{n+2}{n-1}\right)(k-2+\varepsilon(R)) \frac{\left|C_{1}\right|+\cdots+\left|C_{n}\right|}{\left|C_{i_{1}}\right|+\cdots+\left|C_{i_{\frac{n}{2}}}\right|} \\
& \leq \frac{1}{4} \frac{n+2}{n-1}(k-2+\varepsilon(R)) \frac{1}{\frac{1}{2}-\varepsilon(n)} \rightarrow \frac{k-2}{2} .
\end{aligned}
$$

This ends the proof of Theorem 3.1.

q.e.d.

We close this section with a collection of examples of graphs whose Cheeger constant can be shown to be large by elementary methods. We omit the proofs, except for a few comments.

Example 3.1. Let $X_{k}$ be the complete graph of degree $k$, so that $\left|X_{k}\right|=k+1$. Then

$$
h\left(X_{k}\right)=k+1-\left[\frac{k+1}{2}\right] \geq \frac{k+1}{2} .
$$

Similarly, if $Y_{k}$ is the complete bipartite graph of degree $k$, so that $\left|Y_{k}\right|=2 k$, then

$$
h\left(Y_{k}\right)=\frac{k}{2} .
$$

Example 3.2. For $q$ a prime or prime power, we define the incidence graph $P_{q}$ of the projective plane $\mathbb{P}^{2}\left(\mathbb{F}_{q}\right)$ as follows: the vertices of $P_{q}$ are the points and lines of $\mathbb{P}^{2}\left(\mathbb{F}_{q}\right)$, and a point vertex is joined to a line vertex if the corresponding point lies on the corresponding line.

The graph $P_{q}$ has degree $q+1$ and has $2\left(q^{2}+q+1\right)$ vertices.

Then

$$
h\left(P_{q}\right) \geq \frac{q+1}{3} .
$$

Example 3.3. For $q$ a prime, let $\pi_{q}$ be the $q$-th Platonic graph of [7]. This is the graph whose vertices are the pairs $(a, b)$ in $\mathbb{Z} / p \times \mathbb{Z} / p-(0.0)$ with $(a, b)$ identified with $(-a,-b)$, and where $(a, b)$ is joined to $(c, d)$ by an edge if and only if

$$
\operatorname{det}\left(\begin{array}{ll}
a & b \\
c & d
\end{array}\right) \equiv \pm 1(\bmod q) \text {. }
$$


$\pi_{q}$ is $q$-regular on $\frac{q^{2}-1}{2}$ vertices. Then

$$
h\left(\pi_{q}\right) \geq \frac{q^{2}-2 q+5}{4(q-1)} .
$$

The common idea in the above examples is that, given two vertices, one can describe readily the short paths joining them. If the vertices are sent to two sets, then one edge for each short path must be deleted, and one may control how often the same edge is counted.

\section{Relation between the isoperimetric constant and $\lambda_{1}$}

In this section, we study the relationship between the isoperimetric constant $h(X)$ of a graph $X$ and the eigenvalues $\lambda_{0}(X)$ and $\lambda_{1}(X)$ of $\Delta$ acting on $L^{2}(X)$. This is the content of Propositions 4.1 and 4.2. P. Doyle and K. Fujiwara told us that they were aware of Proposition 4.2 .

Proposition 4.1. For a finite graph $X$ of degree $k$ one has

$$
h(X) \geq \frac{1}{2} k \cdot \lambda_{1}(X) .
$$

For an infinite graph $X$ of degree $k$ one has

$$
h(X) \geq k \cdot \lambda_{0}(X) .
$$

This is proved by building test functions out of a division of $X$ by a set realizing $h$.

It is known that:

1. (Kesten [13]). For a regular tree $T_{k}$ of degree $k$,

$$
\lambda_{0}\left(T_{k}\right)=1-\frac{2 \sqrt{k-1}}{k} .
$$

2. For the complete graph $X_{k}$ of degree $k$ one has

$$
\lambda_{1}\left(X_{k}\right)=1+\frac{1}{k}
$$

while for the complete bipartite graph $Y_{k}$ of degree $k$, we have

$$
\lambda_{1}\left(Y_{k}\right)=1-\frac{1}{k} .
$$


3. (Feit-Higman [11]). For the incidence graph $P_{q}$ of a finite projective plane $\mathbb{P}^{2}\left(\mathbb{F}_{q}\right)$ one has

$$
\lambda_{1}\left(P_{q}\right)=1-\frac{\sqrt{q}}{q+1} .
$$

4. The Platonic graph $\pi_{3}$ is the complete graph of degree 3 , and for $q \geq 4$ we have

$$
\lambda_{1}\left(\pi_{q}\right)=1-\frac{1}{\sqrt{q}}
$$

This gives the following estimates for the isoperimetric constants:

1. $h\left(T_{k}\right) \geq k-2 \sqrt{k-1}$.

2. $h\left(X_{k}\right) \geq \frac{k+1}{2}$ and $h\left(Y_{k}\right) \geq \frac{k-1}{2}$.

3. $h\left(P_{q}\right) \geq \frac{q+1-\sqrt{q}}{2}$.

4. $h\left(\pi_{q}\right) \geq \frac{q-\sqrt{q}}{2}$.

Of these inequalities, the first two are weaker than the value found by elementary arguments in Section 3, while for the remaining examples, the bound found here is sharper.

Proposition 4.2. For a finite graph $X$ of degree $k$ one has

$$
h(X) \leq k \sqrt{\lambda_{1}\left(2-\lambda_{1}\right)} .
$$

For an infinite graph $X$ of degree $k$ one has

$$
h(X) \leq k \sqrt{\lambda_{0}\left(2-\lambda_{0}\right)} .
$$

A proof in the case of finite graphs can be found in [10]. The proof for infinite graphs is similar.

Remarks. For a regular tree $T_{k}$ of degree $k$ we have $h\left(T_{k}\right)=k-2$. Proposition 4.2 gives in this case the lower bound

$$
\lambda_{0}\left(T_{k}\right) \geq 1-\frac{2 \sqrt{k-1}}{k},
$$

which is the exact value of $\lambda_{0}\left(T_{k}\right)$. 


\section{The Cheeger constant of a Riemann surface}

In this section, $S$ will denote a compact Riemann surface of genus $>1$, endowed with its metric of constant curvature -1 .

We wish to find an upper bound for the Cheeger constant $h(S)$ of $S$, by mimicking the probabilistic arguments we used in the context of $k$ regular graphs. That is, we will divide $S$ into smaller plaques, and then choose a division of $S$ into two pieces $A$ and $B$ by randomly assigning each plaque to one of these two pieces.

If $D$ is a domain in $S$, let us denote by $h^{*}(D)$ the isoperimetric ratio

$$
h^{*}(D)=\frac{\operatorname{length}(\partial D)}{\operatorname{vol}(D)} .
$$

We denote this ratio by $h^{*}$ to distinguish it from the Cheeger constant $h(D) . h(D)$ and $h^{*}(D)$ are related by

$$
h(D)=\inf _{D^{\prime}} h^{*}\left(D^{\prime}\right)
$$

where $D^{\prime}$ ranges over subdomains of $D$.

A fundamental problem not encountered in the graph-theoretic problem is that the analogue of Lemma 3.1 is no longer true - that is, it is no longer true that a simply-connected domain $D$ in the hyperbolic plane satisfies an upper bound for the ratio $h^{*}(D)$ which is close to the isoperimetric constant of the hyperbolic plane. It is clear that if $D$ contains a ball $B(r)$ of radius $r$, then

$$
h(D) \leq h(B(r))=h^{*}(B(r)),
$$

but $h^{*}(D)$ may be badly behaved for a number of reasons.

In the first place, the boundary of $D$ may be very irregular, and hence long, without greatly affecting the enclosed area. Secondly, even if the boundary of $D$ is very regular, for instance piecewise geodesic, the corners of $D$ will be very inefficient for the isoperimetric ratio $h^{*}$. Thus, an ideal hyperbolic triangle $T$ has $h^{*}(T)=\infty$, since the sides are infinitely long, and if we denote by $D_{n}$ the regular hyperbolic $n$-gon with angles equal to $2 \pi / 3$, we have

$$
h^{*}\left(D_{n}\right) \rightarrow \frac{\operatorname{arccosh}(5 / 3)}{(\pi / 3)}=(3 / \pi) \log (3) \sim 1.049 \cdots>1
$$


as $n \rightarrow \infty$. In particular, if we divide $S$ into plaques $P_{i}$ by placing points $\left\{x_{i}\right\}$ on $S$ and setting

$$
P_{i}=\left\{x \in S: \operatorname{dist}\left(x, x_{i}\right)<\operatorname{dist}\left(x, x_{j}\right), j \neq i\right\},
$$

as we did in the graph-theoretic case, we would not expect in general that $h^{*}\left(P_{i}\right)$ is close to 1 .

We will consider the following method for circumventing this problem: for each number $v$ and integer $k$ such that $k v \leq \operatorname{vol}(S)$, we consider the problem of choosing $k$ disjoint domains $D_{i}$ in $S$ such that for each $i, \operatorname{vol}\left(D_{i}\right)=v$, and so that the value of $\sum_{i}\left(\operatorname{length}\left(\partial D_{i}\right)\right)$ is minimized.

When $v$ is small, this infimum is realized by $k$ disjoint balls of the same radius. When $k v=\operatorname{vol}(S)$, this infimum is realized by $D_{i}$ which are polygonal regions, whose sides are arcs of constant mean curvature, such that precisely three such polygonal regions meet at a vertex [5]. When $k v$ is slightly smaller than $\operatorname{vol}(S)$, the regions $D_{i}$ have boundaries which have piecewise constant curvature, and the set $S \backslash \cup_{i} D_{i}$ consists of triangular regions whose boundaries are constant curvature arcs, the curvature being the value of $h^{*}\left(D_{i}\right)$. These are the "wet soap bubbles" of $[5]$.

We now make the assumption that the injectivity radius of $S$ is large compared to $\operatorname{vol}(S) / k$, and consider the problem of the value of $\max \left(h^{*}\left(D_{i}\right)\right)$ as $v \rightarrow \operatorname{vol}(S) / k$. When $r$ is less than the injectivity radius of $S$ and

$$
\frac{\operatorname{vol}(S)}{\operatorname{vol}(B(2 r))}>k,
$$

we can place in $S k$ disjoint balls of radius $r$, showing that for this value of $r$, and hence for the value $v^{*}=\operatorname{vol}\left(B\left(r^{*}\right)\right)$ for which $h^{*}\left(D_{i}\right)$ reaches its minimum, we have that $h^{*}\left(D_{i}\right)<1+\varepsilon$.

We further make the assumption that for this value of $v^{*}$, the set $S \backslash \cup_{i}\left(D_{i}\right)$ consists only of triangular regions.

Theorem 5.1. With these assumptions, we have that

$$
h(S) \leq(1 / 2)[1+\varepsilon(\operatorname{inj}(S))] .
$$

Proof. We consider the following procedure for dividing $S$ into two pieces $A$ and $B$ : first, we randomly assign each $D_{i}$ to one of the two sets $A$ and $B$. We then have to decide where to place the regions complementary to $\cup_{i}\left(D_{i}\right)$. If $\mathcal{T}$ is such a region, we look at the three regions $D_{i}$ bounding $\mathcal{T}$. If the majority of the $D_{i}$ bounding $\mathcal{T}$ is sent to 
$A$, then we send $\mathcal{T}$ to $A$ as well, and similarly if the majority of the $D_{i}$ are sent to $B$.

We now consider the problem of estimating the isoperimetric constant $h^{*}(A)$. For this, we assume that $k$ is large.

By the Law of Large Numbers, it is clear that approximately half the volume of $\cup_{i}\left(D_{i}\right)$ is sent to $A$. Similarly, for each component $\mathcal{T}$ of $S \backslash \cup_{i}\left(D_{i}\right)$, half the time $\mathcal{T}$ is sent to $A$ and half the time to $B$. So with high probability the value of $\operatorname{vol}(A)$ is close to $(1 / 2) \operatorname{vol}(S)$.

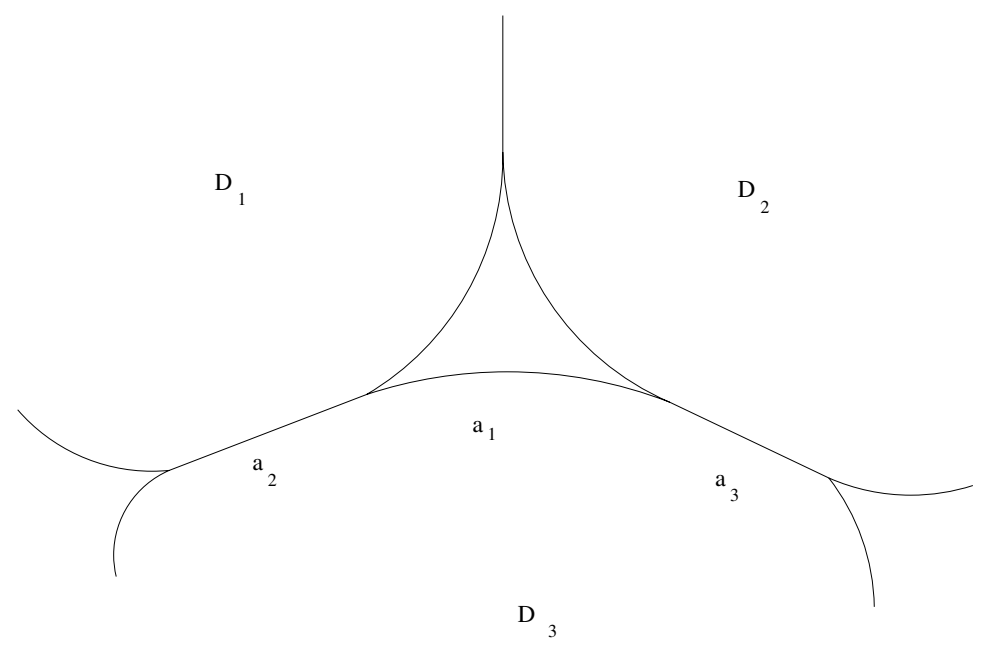

Figure 1: A triangular domain bounded by 3 regions.

We now consider the length of the boundary $\partial(A)$ of $A$. It is clear that the boundary of $A$ is contained in the union of the boundaries of the $D_{i}$ 's. Furthermore, the boundary of each $D_{i}$ consists of arcs $a$ of two types (see Figure 1). Either:

(i) $a$ is the common boundary of two $D_{i}$ 's,

or

(ii) $a$ is the boundary of a triangular region $\mathcal{T}$.

In Case (i), $a$ appears in the boundary of $A$ if and only if one of the $D_{i}$ 's for which it is the common boundary is sent to $A$ while the other is sent to $B$. This happens with probability $1 / 2$.

In Case (ii), $a$ occurs in $\partial A$ if and only if, of the three regions $D_{i}$ bounding $\mathcal{T}$, the one containing $a$ is sent to one set, and the other two are sent to the other set. This occurs with probability $1 / 4$. 
Since in Case (i), $a$ already appears twice, the expected contribution of length $(a)$ to length $(\partial A)$ is $1 / 4$ its contribution to $\sum_{i} \operatorname{length}\left(D_{i}\right)$. So in both Cases (i) and (ii), the expected contribution of a boundary piece $a$ to length $(\partial A)$ is $1 / 4$ its contribution to $\sum_{i} \operatorname{length}\left(D_{i}\right)$.

It follows that with high probability, we have

$$
\begin{aligned}
h^{*}(A) & \sim \frac{(1 / 4) \sum_{i} \operatorname{length}\left(D_{i}\right)}{(1 / 2) \operatorname{vol}(S)} \\
& \leq(1 / 2) \frac{\sum_{i} \operatorname{length}\left(D_{i}\right)}{\sum_{i} \operatorname{vol}\left(D_{i}\right)} \\
& \leq(1 / 2) \max \left(h^{*}\left(D_{i}\right)\right) \leq(1 / 2)(1+\varepsilon) .
\end{aligned}
$$

This concludes the proof of the theorem.

We remark that we get a weaker bound if we assume that complementary regions may be more complicated than triangles. If we assume that each complementary region has at most $m$ sides, the same argument gives the estimate

$$
h^{*}(A) \leq 1-\left(\begin{array}{c}
2 l \\
l
\end{array}\right)\left(\frac{1}{2^{2 l}}\right)
$$

for $m$ equal to either $2 l$ or $2 l+1$.

Note that this expression tends to 1 as $l \rightarrow \infty$.

\section{Modular surfaces}

Let $\Gamma$ denote the group $\operatorname{PSL}(2, \mathbb{Z})$, and $\Gamma_{k}$ the $k$-th congruence subgroup

$$
\Gamma_{k}=\left\{\left(\begin{array}{cc}
a & b \\
c & d
\end{array}\right) \in \Gamma:\left(\begin{array}{cc}
a & b \\
c & d
\end{array}\right) \equiv \pm\left(\begin{array}{cc}
1 & 0 \\
0 & 1
\end{array}\right)(\bmod k)\right\} .
$$

Denote by $S_{k}$ the modular surface

$$
S_{k}=\mathbb{H}^{2} / \Gamma_{k}
$$

$S_{k}$ is a Riemann surface of finite area.

According to a theorem of Selberg, we have

Theorem 6.1 ([20]). $\lambda_{1}\left(S_{k}\right) \geq 3 / 16$. 
Selberg further conjectured that $\lambda_{1}\left(S_{k}\right) \geq 1 / 4=\lambda_{0}\left(\mathbb{H}^{2}\right)$. See $[16]$ for improvements on the $3 / 16$ bound.

In view of the results of $\S 5$, the natural analogue of Selberg's conjecture would be that $h\left(S_{k}\right) \geq 1 / 2$.

In [7], we showed that for all values of $k$, one had the upper bound

$$
h\left(S_{k}\right) \leq .52455 \ldots
$$

and indeed for small values of $k, h\left(S_{k}\right)$ drops below $1 / 2$.

In this section, we apply our probabilistic techniques to a variation of the argument of [7] to show:

Theorem 6.2. There exists a constant $C<1 / 2$ such that

$$
\limsup _{k \rightarrow \infty} h\left(S_{k}\right)<C
$$

We begin the proof in a manner similar to that in [7]. We pick the fundamental domain $F$ for $\Gamma$ shown in Figure 2. We then tile $S_{k}$ by copies of $F$, so that at each cusp, $k$ copies of $F$ meet to form a plaque $P_{k}$, which may be thought of as a regular hyperbolic $k$-gon with all angles equal to $2 \pi / 3$, and a cusp at the center.

As a preliminary step, we consider dividing $S_{k}$ into two sets $A$ and $B$ which are a union of plaques. In [7], we considered a specific way of doing this based on quadratic residues, but now we will simply choose this division randomly.

Denoting by $\mathbf{h}$ the isoperimetric ratio

$$
\mathbf{h}=\frac{\operatorname{length}(\partial A)}{\operatorname{area}(A)},
$$

we may readily compute the expected value of $\mathbf{h}$ as follows: For a given copy of $F$, the probability of its inclusion in $A$ is $1 / 2$. If we denote by $\sigma$ one of the two finite geodesic segments in the boundary of $F$, it will be included in the boundary of $A$ if and only if one of the two copies of $F$ lies in $A$ while the other lies in $B$. This happens with probability $1 / 2$.

Furthermore, each copy of $F$ contributes two copies of $\sigma$, while one copy of $\sigma$ is contributed by two copies of $F$. So

$$
E(h)=\frac{\operatorname{length}(\sigma)}{\operatorname{area}(F)},
$$

which gives as upper estimate for $h\left(S_{k}\right)$ slightly larger than the estimate of [7]. 


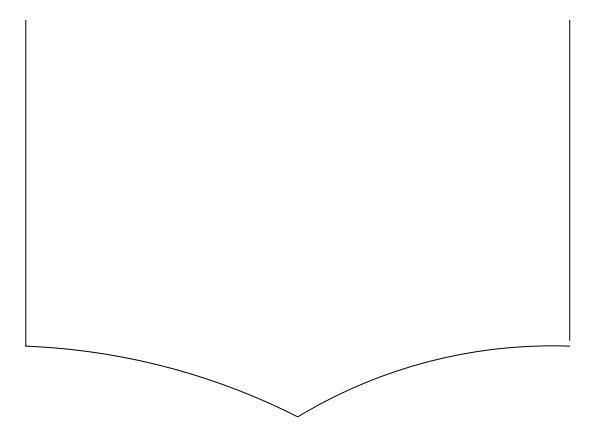

Figure 2: The fundamental domain $F$.

To improve this estimate, we modify the border of $A$ in the following way: for each copy of the vertex of $F$, if two copies of $\sigma$ meet at $v$, we can replace them by the geodesic joining their endpoints. See Figure 3 for this argument.

This will change the area of $A$, since the geodesic cuts off a different piece of the fundamental domain than the two segments, but since half the time this decreases the area of $A$ and half the time it increases the area of $A$, this does not change the expected value of area $(A)$.

On the other hand, the expected value of $\partial A$ is lowered by replacing the length of $\sigma$ by half the length of the geodesic joining $i$ and $i+1$, which has length $\operatorname{arccosh}(3 / 2)$. Denoting by $\mathbf{h}^{\prime}$ the isoperimetric ratio 


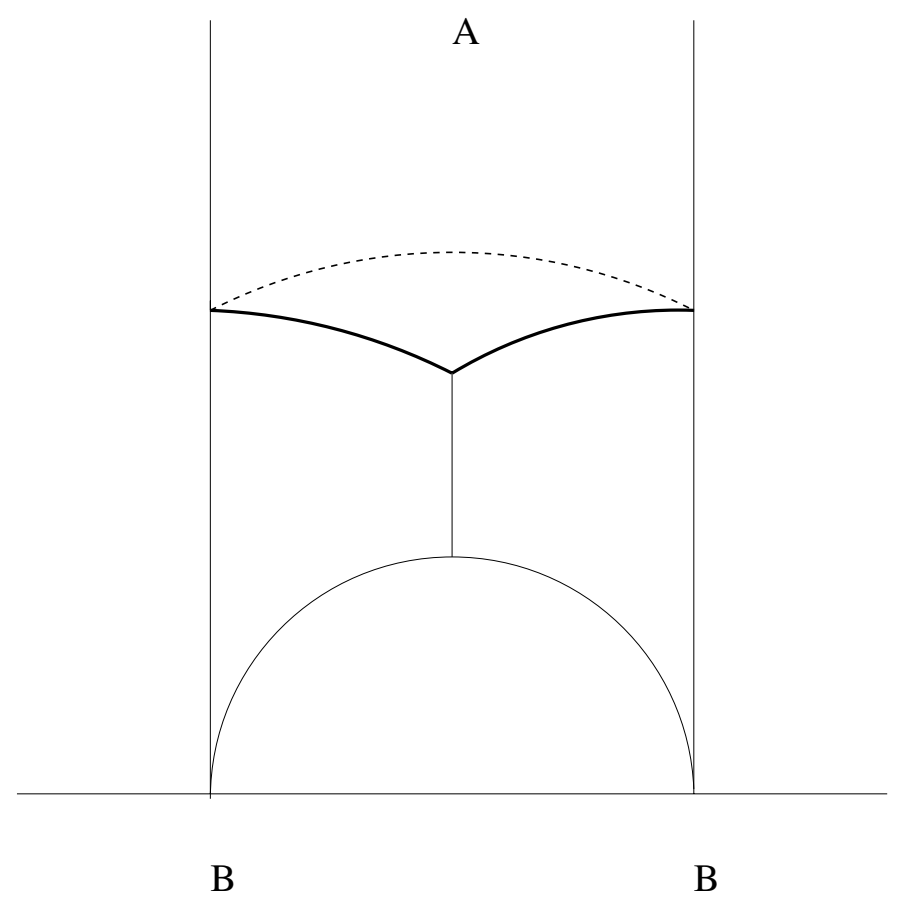

Figure 3: Replacing two geodesic segments by a geodesic.

corresponding to this division, we may modify Equation (2) to obtain

$$
\begin{aligned}
E\left(\mathbf{h}^{\prime}\right) & =\frac{(1 / 2) \operatorname{arccosh}(3 / 2)}{\operatorname{area}(F)} \\
& =\frac{3 \operatorname{arccosh}(3 / 2)}{2 \pi} \\
& \sim 0.4595 .
\end{aligned}
$$

This proves Theorem 6.2 with a value of $C$ of $\frac{3 \operatorname{arccosh}(3 / 2)}{2 \pi} \sim 0.4595$.

We may improve this estimate still further by taking into account that when a section of the boundary of $A$ consists of $n$ geodesic pieces of length $\operatorname{arccosh}(3 / 2)$ isometric to the $n$ geodesic $\operatorname{arcs}(i, i+1),(i+1, i+2)$, $\ldots,(i+(n-1), i+n)$, we may replace it by a geodesic piece joining the two extreme endpoints. When this happens, we may replace the term $(1 / 2) \operatorname{arccosh}(3 / 2)$ by the term $(1 / 2 n) \operatorname{arccosh}\left(1+n^{2} / 2\right)$.

We carry out this argument for the case $n=2$, see Figure 4 for an illustration. In this case, we need only take into account whether the four cusps nearest the geodesic piece are sent to $A$ or $B$. We thus obtain 


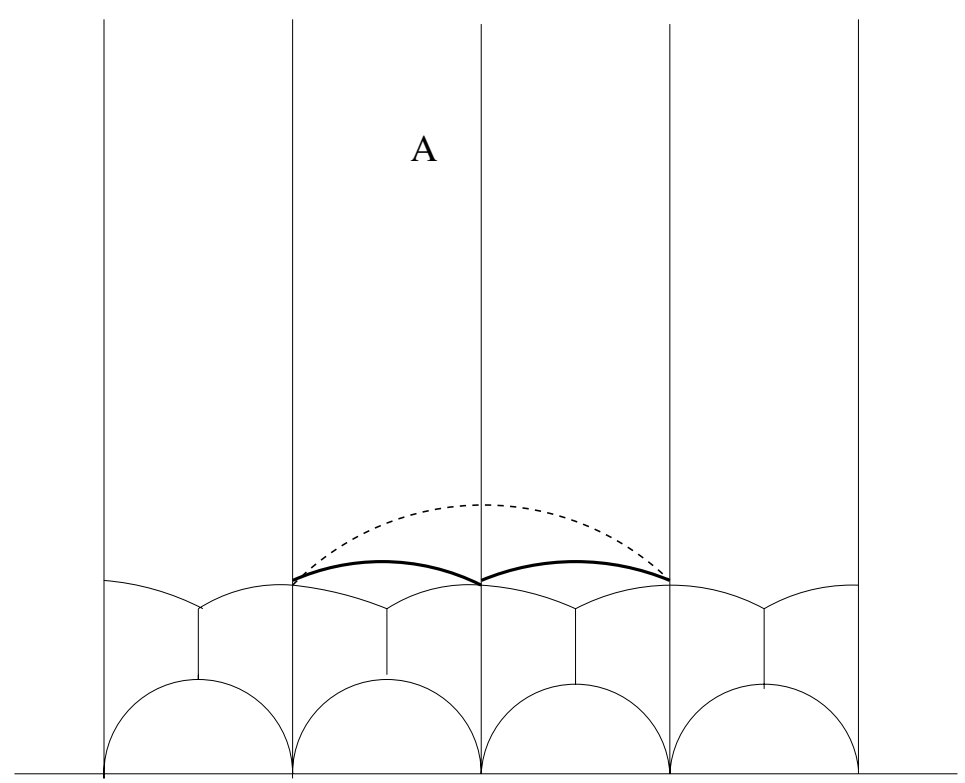

B $\quad$ B $\quad$ B

Figure 4: Replacing two geodesic segments of length $\operatorname{arccosh}(3 / 2)$ by one of length $\operatorname{arccosh}(3)$.

an estimate for $C$ of

$$
C<\frac{3}{8 \pi}[\operatorname{arccosh}(3)+2 \operatorname{arccosh}(3 / 2)] \sim 0.4402 .
$$

\section{Ramanujan surfaces}

We begin this section with a construction taken from [9], which in turn is a generalization of the construction of [8].

If $\Gamma$ is a $k$-regular graph, an orientation $\mathcal{O}$ on $\Gamma$ is an assignment, for each vertex $v$ of $\Gamma$, of a cyclic ordering of the edges emanating from $v$. To each pair $(\Gamma, \mathcal{O})$, we may assign a Riemann surface $S^{O}(\Gamma, \mathcal{O})$ as follows: to each vertex of $\Gamma$, we associate a regular ideal $k$-gon, with a geodesic drawn from the center of the $k$-gon to the midpoint of each side. We identify these geodesics with the edges emanating from each vertex, so that the natural cyclic ordering on the geodesics agrees with the orientation of the graph at the vertex. We then glue two sides 
together so that the midpoints are identified if they correspond to the same edge of the graph (from different vertices).

We denote by $S^{C}(\Gamma, \mathcal{O})$ the conformal compactification of $S^{O}(\Gamma, \mathcal{O})$.

We will apply this construction to a family of graphs $X^{p . q}$ considered by Lubotsky, Phillips, and Sarnak [15], which they call the Ramanujan graphs. These graphs depend on two distinct prime numbers $p$ and $q$. They are $(p+1)$-regular graphs which are homogeneous graphs for the group $G=G^{p, q}$, which is either $\operatorname{PSL}(2, \mathbb{Z} / q)$ or $\operatorname{PGL}(2, \mathbb{Z} / q)$, depending on quadratic residue properties of $p$ and $q$.

In order to apply the construction of [9], we need to choose an orientation $\mathcal{O}$ on $X^{p, q}$. We do this by picking a cyclic ordering at one vertex and translating it over the entire graph by homogeneity. When $p=2$, so that $X^{p, q}$ is 3-regular, the choice of orientation does not matter, but for $p>2$, different choices will give rise to different surfaces. We will denote these surfaces by $S_{\mathcal{O}}^{p, q, O}$ and $S_{\mathcal{O}}^{p, q, C}$, and refer to them as Ramanujan surfaces.

When it does not matter whether we consider the open surface or the closed one, we will suppress the $C$ or $O$. For instance, it follows from [6] that the Cheeger constants of $S_{\mathcal{O}}^{p . q, O}$ and $S_{\mathcal{O}}^{p, q, C}$ will be close for $q$ sufficiently large (depending on $p$ ). We will also suppres the orientation $\mathcal{O}$ when $p=2$.

As an example, we consider the graph $X^{2,3}$. When given the homogeneous orientation, it looks like the picture in Figure 5 below, where the orientation is the natural one induced from the plane.

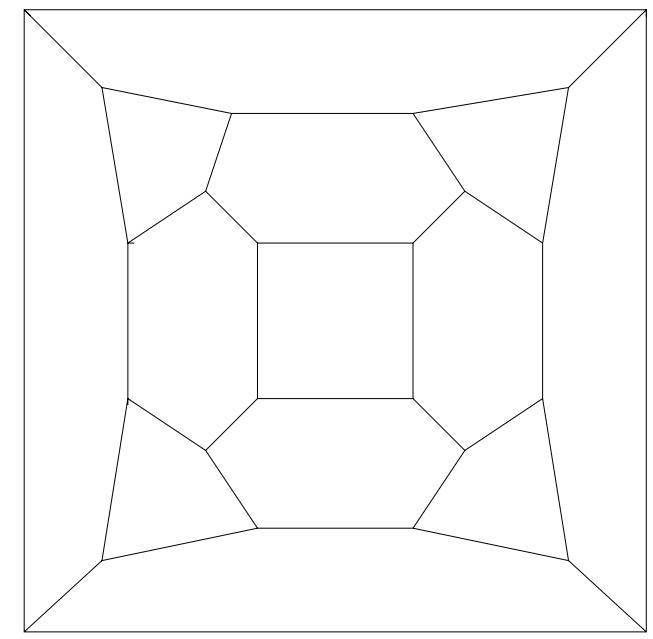

Figure 5: The Ramanujan graph $X^{2,3}$. 
The reader is invited to compare this with the illustration given in [18].

It is clear that $S^{2,3, C}$ is the Riemann sphere. In general, $S_{\mathcal{O}}^{p, q, C}$ is a surface whose Euler characteristic will be given below. It will be negative except for a few exceptional values of $p$ and $q$.

The goal of this section is the following:

Theorem 7.1. There exists a constant $C<1 / 2$ such that, for all $p$, for $q$ sufficiently large (depending on $p$ ), and for all homogeneous $\mathcal{O}$, we have

$$
h\left(S_{\mathcal{O}}^{p, q}\right)<C .
$$

We will find a value of $C$ of $.467177 \ldots$.

Our proof will work equally well for surfaces built from $k$-regular graphs with orientation satisfying certain regularity conditions which will be met for the Ramanujan graphs with a homogeneous orientation. Therefore, we will set $k=p+1$, and consider the case of $k$-regular graphs, $k$ arbitrary.

We will need the following simple facts about the surfaces $S_{\mathcal{O}}^{p, q}$; first of all, the cusps of the surface are obtained by gluing together copies of the fundamental domain $\mathbf{H}_{k}=\mathbf{H}_{p+1}$, shown in Figure 6 below, which is the fundamental domain for the group $H_{k}$ generated by

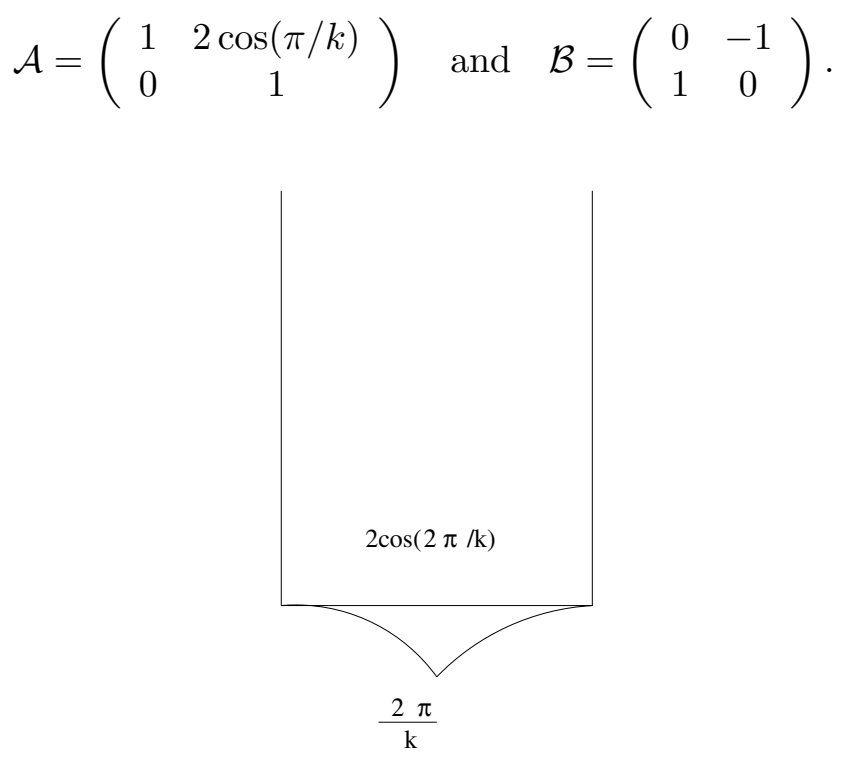

Figure 6: The fundamental domain $\mathbf{H}_{k}$. 
Note that when $k=3$, we obtain the fundamental domain for $\operatorname{PSL}(2, \mathbb{Z})$, while as $k \rightarrow \infty$, the length $2 \cos (\pi / k)$ of the horocycle tends to 2 .

We now claim that there are integers $a_{1}, \ldots, a_{k}$, some of which may agree, such that each cusp of $S_{\mathcal{O}}^{p, q, O}$ is formed by $a_{j}$ copies of the fundamental domain for some $j$, and so that at each vertex, there is one cusp formed from each $a_{j}$ which meets at that vertex. To see that, we recall that since the graph $X^{p, q}$ is the Cayley graph of the group $G^{p, q}$, each edge is labeled by elements $g_{i}$ of the generators of the group. Passing along two edges in succession in the orientation corresponds to multiplying $g_{i}^{-1}$ and $g_{i+1}$. If $g_{i}^{-1}=g_{i+1}$, then the length of the path going around the cusp will be the order of $g_{i}$ in $G^{p, q}$. Otherwise, the length of the path around the cusp will be the order of $2 g_{i+1} g_{i}^{-1}$.

It follows that each $a_{i}$ is at most twice the order of an element of $G^{p, q}$, that is, $a_{i}$ must divide one of the numbers $2 q, 2(q-1)$ or $2(q+1)$. Furthermore, it follows from results of [15] that the girth of $X^{p, q} \rightarrow \infty$ as $q \rightarrow \infty$ (depending on $p$ ), so that the $a_{i}$ 's will also tend to infinity as $q \rightarrow$ $\infty$ (depending on $p$ ). Furthermore, the number of cusps corresponding to $a_{i}$ will be $\left|G^{p, q}\right| / a_{i}$, which will be at least $\frac{(q)(q-1)}{4}$.

In the case of the graph $X^{2,3}$, we see that $a_{1}=4$ while $a_{2}=a_{3}=6$. Note that the Euler characteristic of $S_{\mathcal{O}}^{p, q, C}$ is given by

$$
\chi\left(S_{\mathcal{O}}^{p, q, C}\right)=\left|G^{p, q}\right|\left[\sum_{i=1}^{p+1} \frac{1}{a_{i}}-\frac{p-1}{2}\right],
$$

When $k=3$, we may repeat the argument of Section 6 without change, applying the Law of Large Numbers separately to the groups of cusps of type $a_{i}$ for $i=1,2$, and 3 , to arrive at the same upper bound for $h$ found there.

We now have to consider the cases $k>3$. Here, we are helped by the fact that the horocycles are longer (so that replacing them by geodesics gives greater savings), but are hampered by the fact that the domains outside the horocycles are bounded by more than three sides, and are in fact $k$-sided. We must balance these two tendencies in a way that will produce a uniform upper bound for $h$.

Let $C(k)$ denote the upper bound for $h$ that we get for each $k$. 
We first record some simple facts which we will need. The area of $\mathbf{H}_{k}$ is given by

$$
\operatorname{area}\left(\mathbf{H}_{k}\right)=\pi\left(\frac{k-2}{k}\right)
$$

If $z_{1}$ and $z_{2}$ are two points in the upper half-plane $\mathbb{H}$, then the length $\operatorname{dist}\left(z_{1}, z_{2}\right)$ of the geodesic joining them is given by

$$
\operatorname{dist}\left(z_{1}, z_{2}\right)=\operatorname{arccosh}\left(1+\frac{\left\|z_{2}-z_{1}\right\|^{2}}{2 \operatorname{Im}\left(z_{1}\right) \operatorname{Im}\left(z_{2}\right)}\right),
$$

where $\left\|z_{2}-z_{1}\right\|$ denotes the Euclidean distance.

We now consider the linear fractional transformation

$$
\mathcal{C}=\mathcal{A B}=\left(\begin{array}{cc}
2 \cos (\pi / k) & -1 \\
1 & 0
\end{array}\right)
$$

which is rotation through angle $2 \pi / k$ about the vertex, and define the functions

$$
a_{j}(k)=\operatorname{dist}\left(i, \mathcal{C}^{j}(i)\right) .
$$

$a_{j}(k)$ is the length of the geodesic which spans the endpoints of $j$ horocycles corresponding to $j$ copies of $\mathbf{H}_{k}$ coming together at a vertex.

We have the following formulas for low values of $j$ :

Lemma 7.1. $a_{j}(k)$ is given by the following formulas:

(1) $a_{1}(k)=\operatorname{arccosh}\left(1+2 \cos ^{2}(\pi / k)\right)$.

(2) $a_{2}(k)=\operatorname{arccosh}\left(1+8 \cos ^{4}(\pi / k)\right)$.

(3) $a_{3}(k)=\operatorname{arccosh}\left(1+2 \cos ^{2}(\pi / k)\left[4 \cos ^{2}(\pi / k)-1\right]^{2}\right)$.

(4) $a_{4}(k)=\operatorname{arccosh}\left(1+32 \cos ^{4}(\pi / k)\left[2 \cos ^{2}(\pi / k)-1\right]^{2}\right)$.

(5) $a_{5}(k)=\operatorname{arccosh}\left(1+2 \cos ^{2}(\pi / k)\left[16 \cos ^{4}(\pi / k)\right.\right.$

$$
\left.\left.-12 \cos ^{2}(\pi / k)+1\right]^{2}\right) \text {. }
$$

(6) For all $j$, as $k \rightarrow \infty, a_{j}(k) \rightarrow \operatorname{arccosh}\left(1+2 j^{2}\right)$. 
Proof. This follows from the computations

$$
\begin{gathered}
\mathcal{C}(i)=2 \cos (\pi / k)+1, \quad \mathcal{C}^{2}(i)=\frac{8 \cos ^{3}(\pi / k)+i}{4 \cos ^{2}(\pi / k)+1}, \\
C^{-1}(i)=\frac{2 \cos (p i / k)+i}{4 \cos ^{2}(\pi / k)+1} \\
\mathcal{C}^{3}(i)=\frac{32 \cos ^{5}(\pi / k)-16 \cos ^{3}(\pi / k)+2 \cos (\pi / k)+i}{16 \cos ^{4}(\pi / k)-4 \cos ^{2}(\pi / k)+1}, \\
\mathcal{C}^{-2}(i)=\frac{8 \cos ^{3}(\pi / k)+i}{16 \cos ^{4}(\pi / k)-4 \cos ^{2}(\pi / k)+1}
\end{gathered}
$$

and the distance formula.

q.e.d.

Using these facts, we may now show:

Lemma 7.2. For $k=4,5,6,7$, and 8, we have:

(a) $C(4) \leq .454037001 \ldots$

(b) $C(5) \leq .438076 \ldots$

(c) $C(6) \leq .439071 \ldots$

(d) $C(7) \leq .429074 \ldots$

(e) $C(8) \leq .403043 \ldots$

We first consider the case $k=4$, the other cases being similar. We consider the contributions to the boundary length and area coming from each vertex. For the area term, we have four copies of $\mathbf{H}_{4}$ coming together at the vertex, each of area $\pi / 2$, and expect half of them to contribute to the area term. Hence the contribution to the area at each vertex is $\pi$.

The contribution of the boundary term is more involved. With probability $1 / 8$, all copies of $\mathbf{H}_{4}$ will go to the same set, so there is no boundary. With probability $4 / 8=1 / 2$, one copy will go to one set and the remaining three copies will go to the other set, giving a contribution of $(1 / 2) a_{1}$ to the boundary. Enumerating the remaining possibilities, we see that the expected contribution to the boundary is

$$
\begin{aligned}
& (1 / 8) \cdot 0+(4 / 8) \cdot \alpha_{1}(4)+(1 / 8) \cdot 2 \alpha_{1}(4)+(1 / 4) \cdot \alpha_{2}(4) \\
& =(3 / 4) \alpha_{1}(4)+(1 / 4) \alpha_{2}(4) \\
& =(3 / 4) \operatorname{arccosh}(3)+(1 / 4) \operatorname{arccosh}(3) \\
& =1.42871 \ldots,
\end{aligned}
$$


and dividing by $\pi$ gives our estimate for $C(4)$.

In general, the contribution to the area at a vertex will be $\pi\left(\frac{k-2}{2}\right)$, so the problem will be in calculating the contribution $b(k)$ to the boundary. Counting the possibilities as above gives

$$
\begin{aligned}
& b(5)=(5 / 16)\left[3 \alpha_{1}(5)+\alpha_{2}(5)\right] . \\
& b(6)=(1 / 32)\left[33 \alpha_{1}(6)+12 \alpha_{2}(6)+3 \alpha_{3}(6)\right] . \\
& b(7)=(7 / 64)\left[9 \alpha_{1}(7)+3 \alpha_{2}(7)+\alpha_{3}(7)\right] . \\
& b(8)=(1 / 32)\left[37 \alpha_{1}(8)+16 \alpha_{2}(8)+5 \alpha_{3}(8)+\alpha_{4}(8)\right] .
\end{aligned}
$$

Plugging these formulas into a calculator then gives the lemma.

We clearly need a different idea for large $k$. We will adopt the following: given a copy of $\mathbf{H}_{k}$, we calculate the probability that it is part of a group of $j$ adjoining fundamental domains, each of which is sent to the same set. If in addition this copy belongs to the minority, then the contribution to the boundary will be

$$
\frac{1}{2}\left[\frac{a_{j}(k)}{j}\right] .
$$

This is clearly an overestimate, since if a copy is surrounded by a large number of domains going to the same set, this increases the probability that it belongs to the majority. But this effect is small when $k$ is large.

We now claim:

Lemma 7.3. The function $\frac{a_{j}(k)}{j}$ is a decreasing function of $j$ for $j \leq k$.

Proof. Let $D_{\theta}$ be a rotation through angle $2 \theta$ about some fixed point. For instance, we may take

$$
D_{\theta}(z)=\left(\begin{array}{cc}
\cos (\theta) & \sin (\theta) \\
-\sin (\theta) & \cos (\theta)
\end{array}\right)
$$

and let $d_{z_{1}}$ be the function of $\theta$ given by

$$
d_{z_{1}}(\theta)=\operatorname{dist}\left(z_{1}, D_{\theta\left(z_{1}\right)}\right) \text {. }
$$

Then

$$
d_{z_{1}}(\theta)=\operatorname{arccosh}\left(1+(\text { const }) \sin ^{2}(\theta)\right),
$$


where (const) depends only on the distance of $z_{1}$ to the fixed point.

This can most easily be verified by taking $D_{\theta}$ to be the rotation about 0 in the disk, and using the analogue of the distance formula for the disk.

The assertion of the lemma now follows from the fact that for $\theta \leq$ $\pi / 2$

$$
\frac{d_{z_{1}}(\theta)}{\theta} \text { is a decreasing function of } \theta
$$

which in turn can be verified by calculus.

q.e.d.

We may calculate the probability $P_{l}$ of a given copy of $\mathbf{H}_{k}$ to be part of a block of exactly $l$ copies which are sent to the same set by

$$
P_{l}=\frac{l}{2^{l+1}},
$$

provided $l \leq k-2$. Using Lemma 7.3, we see that for any $m \leq k-2$, the expected contribution at a given copy of $\mathbf{H}_{k}$ is bounded above by

$$
\frac{1}{2}\left[\left(\sum_{l=1}^{m-1} P_{l} \frac{a_{l}}{l}\right)+\left(1-\sum_{l=1}^{m-1} P_{l}\right)\left(\frac{a_{m}}{m}\right)\right] .
$$

Applying this to $m=5$, we have the estimate

$$
\begin{aligned}
C(k) \leq \frac{1}{\pi(1-2 / k)}\left[\frac{1}{4} a_{1}(k)+\right. & \frac{2}{8} \frac{a_{2}(k)}{2} \\
& \left.+\frac{3}{16} \frac{a_{3}(k)}{3}+\frac{4}{32} \frac{a_{4}(k)}{4}+\frac{3}{16} \frac{a_{5}(k)}{5}\right] .
\end{aligned}
$$

In the limit when $k \rightarrow \infty$, we get that this converges to

$$
\begin{aligned}
& \frac{1}{\pi}\left[\frac{\operatorname{arccosh}(3)}{4}+\frac{\operatorname{arccosh}(9)}{8}+\frac{\operatorname{arccosh}(19)}{16}\right. \\
& \left.\quad+\frac{\operatorname{arccosh}(33)}{32}+\frac{3 \operatorname{arccosh}(51)}{80}\right] \\
& =.404434 \ldots
\end{aligned}
$$

Furthermore, this function is decreasing in $k$ for $k>8$, and is always below .467177 after $k=8$.

This completes the proof of the theorem.

q.e.d. 


\section{References}

[1] N. Alon, Eigenvalues and expanders, Combinatorica 6 (1986) 83-96, MR 88e:05077, Zbl 0661.05053.

[2] N. Alon, On the edge-expansion of graphs, Combin. Probab. Comput. 6 (1997) 145-152, MR 98c:05084, Zbl 0881.05077.

[3] B. Bollobás, The isoperimetric number of random regular graphs, European J. Combin. 9(3) (1988) 241-244, MR 89e:05180, Zbl 0673.05086.

[4] B. Bollobás, Random Graphs (2nd edition), Cambridge Studies in Advanced Mathematics, 73, Cambridge University Press, Cambridge, 2001, MR 2002j:05132, Zbl 0979.05003.

[5] K. Brakke \& F. Morgan, Instability of the wet X soap film, J. Geom. Anal. 8 (1998) 749-767, MR 2001f:49075, Zbl 0965.53012.

[6] R. Brooks, Platonic surfaces, Comm. Math. Helv. 74(1999) 156-170, MR 99k:58185, Zbl 0920.30037.

[7] R. Brooks, Spectral geometry and the Cheeger constant, in 'Expanding graphs' (Princeton, NJ, 1992), 5-19, DIMACS Ser. Discrete Math. Theoret. Comput. Sci., 10, Amer. Math. Soc., Providence, RI, 1993, MR 1235 564, Zbl 0788.58055.

[8] R. Brooks \& E. Makover, Random construction of Riemann surfaces, preprint.

[9] R. Brooks \& M. Monastyrsky, in preparation.

[10] F.R.K. Chung, Spectral Graph Theory, CBMS Regional Conference Series in Mathematics, 92, Published for the Conference Board of the Mathematical Sciences, Washington, DC; by the American Mathematical Society, Providence, RI, 1997, MR 97k:58183, Zbl 0867.05046.

[11] W. Feit \& G. Higman, The nonexistence of certain generalized polygons, J. Algebra 1 (1964) 114-131, MR 30 \#1189, Zbl 0126.05303.

[12] W. Feller, An Introduction to the Probability Theory. Vol. II, Wiley and Sons, New York, 1966, MR 35 \#1048, Zbl 0138.10207.

[13] H. Kesten, Symmetric random walks on groups, Trans. Amer. Math. Soc. 92 (1959) 336-354, MR 22 \#253, Zbl 0092.33503.

[14] A. Lubotzky, Discrete Groups, Expanding Graphs and Invariant Measures, Progress in Mathematics, 125, Birkhäuser, 1994, MR 96g:22018, Zbl 0826.22012.

[15] A. Lubotzky, R. Phillips \& P. Sarnak, Ramanujan graphs, Combinatorica 8 (1988) 261-277, MR 89m:05099, Zbl 0661.05035.

[16] W. Luo, Z. Rudnick \& P. Sarnak, On Selberg's eigenvalue conjecture, Geom. Funct. Anal. 5 (1995) 387-401, MR 96h:11045, Zbl 0844.11038. 
[17] G. Margulis, Explicit group theoretic constructions of combinatorial schemes and their applications for the construction of expanders and concentrators, J. of Problems of Information Transmission 24 (1988) 39-46, MR 89f:68054.

[18] P. Sarnak, Some Applications of Modular Forms, Cambridge Tracts in Mathematics, 99, Cambridge University Press, 1990, MR 92k:11045, Zbl 0721.11015.

[19] J.-P. Serre, Répartition asymptotique des valeurs propres de l'opérateur de Hecke $T_{p}$, J. Amer. Math. Soc. 10(1) (1997) 75-102, MR 97h:11048, Zbl 0871.11032.

[20] A. Selberg, On the estimation of Fourier coefficients of modular forms, in 'Theory of Numbers' (A.L. Whiteman, ed.) Proc. Symp. Pure Math 8 (1965) 1-15, MR 32 \#93, Zbl 0142.33903.

Department of Mathematics

Technion, Haifa, IsRael

CNRS, Ecole Normale Supérieure de Lyon F-69364 Lyon CEDEX 07, FranCE 